NBI-HE-93-50

August 1993

\title{
On String Tunneling in Power Law Inflationary Universes.
}

\author{
A.L.Larsen 円 \\ Nordita, 17 Blegdamsvej, 2100 Copenhagen, Denmark \\ Minos Axenides ? \\ The Niels Bohr Institute \\ University of Copenhagen, 17 Blegdamsvej, 2100 Copenhagen, Denmark
}

\begin{abstract}
We consider the evolution of circular string loops in power law expanding universes represented by a spatially flat Friedman-RobertsonWalker metric with scale factor $a(t) \propto t^{p}$, where $t$ is the cosmic time and $p \geq 0$. Our main result is the existence of a "magic" power $p_{m}=3+2 \sqrt{2}$. In spacetimes with $p<p_{m}$ a circular string expands either forever or to a maximal radius and then contracts until it collapses into a point (black hole). For $p \geq p_{m}$, however, we find additional types of solutions. They include configurations which contract from a positive initial radius to a minimal one and then expand forever. Their existence we interpret as an indication for the presence of a finite potential barrier. Equivalently the new solutions signal string nucleation and tunneling, phenomena recently shown to occur in de Sitter space.
\end{abstract}

\footnotetext{
${ }^{1}$ e-mail:allarsen@nbivax.nbi.dk

${ }^{2}$ e-mail:axenides@nbivax.nbi.dk
} 


\section{Introduction}

Inflation [1] is a period of rapid expansion in the early history of the universe. Through its different realizations (power law, intermediate, exponential etc.) many cosmological paradoxes find a simultaneous resolution. Topological defects such as cosmic strings, monopoles and domain walls are typically generated at cosmic phase transitions through the Kibble mechanism [2]. It has been a theoretical prejudice that an unwanted overabundance of such objects in the observable universe can be eliminated by an inflationary phase following such phase transitions. More specifically the cosmic string scenario for the generation of the large scale structure was considered to be hardly compatible with inflation in a single theoretical framework [3].

Recently in a very interesting paper [4] Basu, Guth and Vilenkin took a step towards resolving the conflict by demonstrating the spontaneous nucleation of cosmic strings in de Sitter space. Using the static parametrization they found that the classical evolution of a circular string is determined by a simple potential barrier and that strings can nucleate by a quantum mechanical tunneling through the barrier. The tunneling amplitude that determines the nucleation rate could finally be calculated using either the WKB approximation or the instanton method. String nucleation of ref.(4) has been further investigated in refs. (5-9). The case of charged string tunneling processes in de Sitter space has also appeared in the literature [10].

The purpose of the present work is to consider the possibility of string nucleation in power law inflationary universes [11]. The first problem we meet is that such spacetimes do not have a static parametrization. Therefore we cannot expect that the evolution of classical circular string loops is determined by a simple one dimensional Hamiltonian with a simple potential, from which the tunneling amplitude can be calculated explicitly, as was the case in de Sitter space [4]. Secondly, the equations of motion for the circular strings are not even integrable in the cases under consideration here. We will therefore use more indirect analytical and numerical methods, and will restrict ourselves to formulating only necessary and sufficient conditions on time dependent spacetimes for string nucleation to happen, and moreover argue for its occurence in the case to be considered.

Our starting point is that string nucleation of circular loops is intimately connected to the phenomenon of string tunneling [4]. We therefore expect that string nucleation may play an important role in cosmological spacetimes that admit circular string configurations with the property :

$$
\exists t_{0}: \quad f(t) \geq f\left(t_{0}\right)>0, \quad \frac{d f}{d t}\left(t_{0}\right)=0
$$

where $f$ is the physical radius of the loop and $t$ is the cosmic time. Loosely speaking eq.(1.1) expresses that there are circular string configurations which are "energetically" forbidden to collapse, i.e. if they contract they will sooner 
or later hit a "barrier" (at $t=t_{0}$ ). The existence of such a barrier on the other hand means that tunneling effects may be relevant and that circular strings may nucleate with finite radius. We can also of course imagine strings collapsing by tunneling the other way through the barrier.

In the present paper we will consider time dependent spacetimes and therefore the condition (1.1) must hold only locally around $t_{0}$, i.e. for :

$$
t \in\left[t_{0}-\Delta t_{1}, t_{0}+\Delta t_{2}\right]
$$

for some positive $\Delta t_{1,2}$. Loosely speaking we may say that a barrier that prevents a string from collapsing at $t=t_{0}$ will change in time, and in some cases may only exist for a finite time during the evolution of the universe. This means that a nucleated string which is created by tunneling through a barrier, in a later moment of the cosmic expansion (where the original potential barrier has disappeared again) can collapse by continuously and classically shrinking to a point [12]. We believe the above picture to be realistic and physical as our universe went through a de Sitter like phase in its early moments before it entered the radiation dominated era. In the latter phase the string evolution equations indicate an unconditional string collapse. This is also the physical picture that was recently adopted by Garriga and Vilenkin [8] where de Sitter spacetime is patched next to a Minkowski one as an approximation to the radiation dominated universe. It should be stressed that in what follows we are not directly studying the nucleated strings. Instead we use "test strings" with all kinds of initial values of radii and velocities in order to explore the structure of the interaction between the string and the underlying expanding universe. As explained above, this approach provides information about the possible existence of tunneling phenomena and string nucleation processes.

The paper is organized as follows. In section 2 we derive the equations of motion that determine the classical evolution of circular strings in a spatially flat Friedman-Robertson-Walker(FRW) universe [13, 14, 15, 16], in a gauge where the string time is identified with the cosmic time. In section 3 we analyze the equations of motion in power law expanding universes and find a "magic" power related to tunneling phenomena. We also present numerical results that confirm the significance of this magic power and we discuss some of the string trajectories. Finally in section 4 we present our conclusions.

\section{The equations of motion}

We consider the equations of motion for a circular string in a spatially flat FRW spacetime, in the special case of the scale factor being a power of the cosmic time. We therefore take the line element in the form:

$$
d s^{2}=-d t^{2}+a(t)^{2}\left(d r^{2}+r^{2} d \theta^{2}+r^{2} \sin ^{2} \theta d \phi^{2}\right)
$$


and we will eventually take

$$
a(t)=a_{p} t^{p} ; \quad p \geq 0
$$

where $a_{p}$ is a dimensionful constant which is included to ensure that $a(t)$ is dimensionless. Note that the family of spacetimes parametrized in (2.2) includes Minkowski space $(p=0)$, a radiation dominated universe $(p=1 / 2)$, a matter dominated universe $(p=2 / 3)$, a linearly expanding universe $(p=1)$ and power law inflationary universes $(p>1)$.

Before we embed a circular string let us recall a few facts about these spacetimes. It is the existence of either particle horizons or event horizons [17. For $1>p \geq 0$ there is a particle horizon with a physical radius given by :

$$
f_{P H}(t)=a(t) r_{P H}(t) \equiv a(t) \int_{0}^{t} \frac{d s}{a(s)}=\frac{t}{1-p}
$$

while for $p>1$ there is an event horizon with a physical radius given by:

$$
f_{E H}=a(t) r_{E H}(t) \equiv a(t) \int_{t}^{\infty} \frac{d s}{a(s)}=\frac{t}{p-1}
$$

Both types of horizon will be essential later on in our analysis of the evolution of circular strings. It is also worth keeping in mind the expressions for the nonvanishing components of the Riemann tensor. In cartesian coordinates we have that:

$$
\begin{aligned}
& R_{x t x t}=R_{y t y t}=R_{z t z t}=-a(t) \frac{d^{2} a(t)}{d t^{2}}=-a_{p}^{2} p(p-1) t^{2(p-1)} \\
& R_{x y x y}=R_{x z x z}=R_{y z y z}=a(t)^{2}\left(\frac{d a(t)}{d t}\right)^{2}=a_{p}^{4} p^{2} t^{2(2 p-1)}
\end{aligned}
$$

So spacetimes $(2.1-2.2)$ are plagued with a spacetime singularity at $t=0$ unless either $p=0$ or $p \geq 1$.

Let us now consider a classical string described by the Nambu-Goto action:

$$
\mathcal{L} \sim \sqrt{-\operatorname{det} G_{\alpha \beta}}
$$

where $G_{\alpha \beta}$ is the induced metric on the world-sheet

$$
G_{\alpha \beta}=g_{\mu \nu} X_{, \alpha}^{\mu} X_{, \beta}^{\nu}
$$

with $X^{\mu}=(t, r, \theta, \phi)$ and $g_{\mu \nu}=\operatorname{diag}\left(-1, a^{2}, a^{2} r^{2}, a^{2} r^{2} \sin ^{2} \theta\right)$. For completeness we have kept the scale-factor arbitrary. A circular string is most easily obtained by the following combined gauge choice and Ansatz:

$$
t=\tau, \quad r=r(t), \quad \theta=\pi / 2, \quad \phi=\sigma
$$

where $(\tau, \sigma)$ are the two internal world-sheet coordinates. The equations of motion obtained from the Lagrangian (2.6) and the ansatz (2.8) are: 


$$
\begin{aligned}
& \frac{d}{d t}\left[\frac{a^{2} r^{2}}{\sqrt{a^{2} r^{2}\left(1-a^{2} \dot{r}^{2}\right)}}\right]+\frac{\dot{a} a r^{2}\left(2 a^{2} \dot{r}^{2}-1\right)}{\sqrt{a^{2} r^{2}\left(1-a^{2} \dot{r}^{2}\right)}}=0 \\
& \frac{d}{d t}\left[\frac{a^{4} r^{2} \dot{r}}{\sqrt{a^{2} r^{2}\left(1-a^{2} \dot{r}^{2}\right)}}\right]+\frac{a^{2} r\left(a^{2} \dot{r}^{2}-1\right)}{\sqrt{a^{2} r^{2}\left(1-a^{2} \dot{r}^{2}\right)}}=0
\end{aligned}
$$

where the dot denotes differentiation with respect to $t$. This looks quite complicated but after a little algebra one finds [13, 15 that both equations reduce to:

$$
a^{2} r \ddot{r}-2 r \dot{a} a^{3} \dot{r}^{3}-a^{2} \dot{r}^{2}+3 a \dot{a} r \dot{r}+1=0
$$

For our purposes it is convenient to introduce the physical string radius:

$$
f=a r
$$

and the Hubble function:

$$
H=\frac{\dot{a}}{a}
$$

Equation (2.10) becomes:

$$
\begin{aligned}
\ddot{f} f-2 H f \dot{f}^{3} & +\left(6 H^{2} f^{2}-1\right) \dot{f}^{2}+3 H f\left(1-2 H^{2} f^{2}\right) \dot{f} \\
& -f^{2} \dot{H}+2 H^{4} f^{4}-3 H^{2} f^{2}+1=0
\end{aligned}
$$

The above equation gives the physical string radius $f$ as a function of the cosmic time in a spatially flat FRW spacetime with a Hubble function H. It's exact analytical solution does not seem to be available, but it is very suitable for a numerical investigation. With regard to the boundary conditions we should keep in mind that a timelike string is obtained provided:

$$
G_{00}=-1+a^{2} \dot{r}^{2}=-1+(\dot{f}-H f)^{2}<0
$$

In the rest of our investigation we will look for configurations that satisfy the above inequality. For the special case of $a(t)=a_{p} t^{p}$ and $H(t)=p / t$ eq.(2.13) takes the form:

$\ddot{f} f-\frac{2 p}{t} f \dot{f}^{3}+\left(\frac{6 p^{2}}{t^{2}} f^{2}-1\right) \dot{f}^{2}+\frac{3 p}{t}\left(1-\frac{2 p^{2}}{t^{2}} f^{2}\right) \dot{f} f+\frac{2 p^{4}}{t^{4}} f^{4}+p(1-3 p) \frac{f^{2}}{t^{2}}+1=0$

Both eqs. $(2.13,2.15)$ will be studied in more detail in the next section.

\section{The magic power and numerical solutions}

Let us assume that for some scalefactor $a(t)$ we find a string configuration that satisfies conditions (1.1) and (1.2) at some fixed $t_{0}$. By a Taylor expansion of 
$f(t)$ around $t_{0}$ we get that $\ddot{f}\left(t_{0}\right) \geq 0$. The equation of motion (2.13) for $f(t)$ gives at $t=t_{0}$ :

$$
\dot{H}\left(t_{0}\right) \geq \frac{1}{f\left(t_{0}\right)^{2}}+2 H\left(t_{0}\right)^{4} f\left(t_{0}\right)^{2}-3 H\left(t_{0}\right)^{2}
$$

On the other hand condition (2.14) gives for $t=t_{0}$ :

$$
\frac{1}{H\left(t_{0}\right)}>f\left(t_{0}\right)>0
$$

and by combining (3.1) with (3.2) we find:

$$
\dot{H}\left(t_{0}\right) \geq(2 \sqrt{2}-3) H\left(t_{0}\right)^{2}
$$

According to the discussion of the interpretation of $t_{0}$ (see the introduction) this inequality gives a necessary condition for the existence of string loop tunneling and nucleation. We may note in passing that (3.3) is trivially fulfilled for the de Sitter spacetime $\left(a \sim e^{t}, H=\right.$ const). A remarkable fact about power law expanding universes is that $\dot{H}$ is proportional to $H^{2}$ so that $t_{0}$ drops out of (3.3). In fact from (2.2) we get:

$$
p \geq 3+2 \sqrt{2} \equiv p_{m}
$$

which is exactly the "magic" power advertised in the abstract. If $p<p_{m}$ there can be no string configuration fulfilling conditions $(1.1-1.2)$. This implies that a circular string will either expand monotonically for ever, contract monotonically until it collapses, or expand monotonically to a maximal radius $f(\dot{t})($ where $\dot{f}(\dot{t})=0, \ddot{f}(\dot{t})<0$ ) and then contract monotonically until it collapses. Examples of such configurations obtained by numerically integrating equation (2.15) are shown in figs.1 and 2. It should be remarked that from a mathematical point of view the collapsing strings can be continued below $f=0$ and then appear as oscillating. We, however, take the cosmic string point of view where the evolution of the string stops the first time the physical radius reaches zero from above and the string has collapsed into a black hole [12]. Let us now consider spacetimes where $p \geq p_{m}$, i.e. for which inequality (3.4) is satisfied. In this case we expect to find configurations contracting to a minimal radius, subsequently expanding. By numerically integrating eq.(2.15) we have indeed found such solutions for all $p \geq p_{m}$. We present two such examples in figures 3 and 4 . Note that after bouncing at the barrier the string, depending on its initial radius and velocity, can either continue to expand forever (fig.3) or actually collapses ultimately anyway (fig.4). In addition we found solutions of the form presented in figs.1 and 2. In summary the solutions for $p \geq p_{m}$ include strings bouncing from below (smaller radius), strings bouncing from above (larger radius) and strings monotonically expanding from $f=0$ to $f \rightarrow \infty$. This fact indicates that the classical evolution of strings is determined by a finite "potential barrier" and we therefore expect that string 
tunneling and nucleation take place for spacetimes with $p \geq p_{m}$. We should stress once more however, that we are considering time-dependent spacetimes that do not admit static parametrizations. As a consequence the apparent potential barriers are to be interpreted as being similarly time dependent, in contrast to the case of de Sitter spacetime [ [ 1 ].

\section{Conclusion}

In the present work we explored the possibility that quantum nucleation of topological defects such as cosmic strings, is not a property of the de Sitter spacetime alone. To that end we studied the circular string evolution equations in spacetimes with variable power law expansion rate. Surprisingly, in addition to purely expanding and collapsing solutions, we found a new type of solutions. They occur for spacetimes with a power greater than $3+2 \sqrt{2}$ which we interpreted as evidence for the presence of string nucleation. Quantum tunneling of circular strings appears to occur in almost all types of generalized inflationary cosmologies. A full classification of the different types of circular string evolution in such spacetimes will be the subject of future work. 


\section{References}

[1] A. Linde, 'Particle Physics and Inflationary Cosmology' (Harwood, Chur, 1990).

[2] E.W. Kolb and M.S. Turner, 'The Early Universe' (Addison-Wesley 1989).

[3] R.H. Brandenberger in 'Lectures in Modern Cosmology and Structure Formation', proc. of the 7th Swieca Summer School (World Scientific, Singapore, 1993).

[4] R. Basu, A.H. Guth and A. Vilenkin, Phys. Rev. D 44 (1991) 340.

[5] R. Basu and A. Vilenkin, Phys. Rev. D 46 (1992) 2345.

[6] J. Garriga and A. Vilenkin, Phys. Rev. D 44 (1991) 1007.

[7] J. Garriga and A. Vilenkin, Phys. Rev. D 45 (1992) 3469.

[8] J. Garriga and A. Vilenkin, Phys. Rev. D 47 (1993) 3265.

[9] J. Garriga, hep-ph/9308280.

[10] A. Davidson, N.K. Nielsen and Y. Verbin, Preprint(1992).

[11] F. Luchin and S. Matarrese, Phys. Rev. D 32 (1985) 1316, L.F. Abbot and M.B. Wise, Nucl. Phys. B 244 (1984) 541, D. La and P.J. Steinhardt, Phys. Rev. Lett. 62 (1989) 376, D. La, P.J. Steinhardt and E.W. Bertschinger, Phys. Rev. D 40 (1989) 3950, E.J. Weinberg, Phys. Rev. D 40 (1989) 3950.

[12] S.W. Hawking, Phys. Lett. B 246 (1990) 36.

[13] K.G. Akdeniz, S. Erbil, H. Mutus and E. Rizaoglu, Phys. Lett. B 237 (1990) 192.

[14] A. Vilenkin, Phys. Rev. D 43 (1991) 1060.

[15] X. Li and J. Zhang, Phys. Lett. B 312 (1993) 62.

[16] H.J de Vega and I.L. Egusquiza, PAR-LPTHE-93/43(1993).

[17] W.Rindler, 'Essential Relativity' (Springer-Verlag 1979), 2nd edition, sect.(9.6). 


\section{Figure Captions}

Fig.1. Examples of string trajectories for $p=1 / 2$ (radiation dominated universe). The particle horizon $f_{P H}=2 t$ can only be passed from the outside, and soon after passing it the circular strings collapse [15].

Fig.2. Examples of string trajectories for $p=2$. The presence of an event horizon $f_{E H}=t$ guarantees the existence of non-collapsing solutions: Once outside, the string expands towards infinity. We however also find collapsing solutions in this case. They are of course always inside the event horizon.

Fig.3. Example of a string trajectory for $p=25$, i.e. $p>p_{m}$. The string contracts to a minimal size, then expands through the event horizon $f_{E H}=$ $t / 24$ and continues towards infinity.

Fig.4. Another example of a string trajectory for $p=25$. The string contracts to a local minimal size, then expands to a maximal size inside the event horizon and finally collapses to a point. 